\title{
Évaluation de la qualité physico-chimique de certains tributaires d'eaux usées du lac Ahémé au Bénin
}

\author{
${ }^{1}$ Comlan Achille Dèdjiho*, ${ }^{1}$ Daouda Mama, ${ }^{1}$ Lyde Tomètin, ${ }^{1}$ Irma Nougbodé, ${ }^{1}$ Waris Chouti et ${ }^{2} \mathrm{C}$. K. \\ Dominique Sohounhloué, ${ }^{1}$ Moussa Boukari \\ 1'Laboratoire d'Hydrologie Appliquée (LHA), Faculté des Sciences et Techniques (FAST), Université d'Abomey-Calavi 01 \\ BP : 526 Cotonou, Bénin. \\ 2Laboratoire d'Étude et de Recherche en Chimie Appliquée (LERCA), École Polytechnique d'Abomey-Calavi (EPAC), \\ Université d'Abomey-Calavi, Bénin. \\ *Auteur correspondant : dedjiho1@yahoo.fr Tél : (00229) 95021133 \\ Original submitted in on $6^{\text {th }}$ September Published online at www.m.elewa.org on 31st October 2013.
}

\section{RÉSUMÉ}

Objectif: L'objectif de ce travail mené est d'évaluer la qualité physico-chimique du Lac Ahémé au Bénin afin de connaître les risques possibles pour les espèces aquatiques et la santé humaine.

Méthodologie et résultats : Les méthodes utilisées sont celles spectrophotométriques, colorimétriques et quelques autres recommandées par l'Association Française de Normalisation (AFNOR). Les stations échantillonnées sont révélées pauvres en oxygènes dissous et présentent un état d'eutrophisation.

Conclusion et application : Cette situation doit attirer l'attention des riverains et des décisions politiques sur la gestion des eaux usées et des déchets agricoles, domestiques et industriels.

Mots clés : Qualité physico-chimique, lac Ahémé, eutrophisation, déchets

\begin{abstract}
\section{INTRODUCTION}

Les eaux de surface sont aujourd'hui sujettes à une affreuse agression qui est la pollution. En Corée du Sud, comme en Chine ou en Inde, la qualité des plans s'est drastiquement détériorée (Chang, 2005; He et al, 2007 ; Paliwal et al, 2007). En Afrique, la pollution des plans d'eau est d'actualité et le traitement des eaux usées n'est pas toujours une
\end{abstract}

Objective: The objective of this work is to evaluate the physico-chemical quality of the Lake Ahémé in Benin in order to know the possible risks for the aquatic species and the human health.

Methodology and results : The used methods are those spectrophotométriques, colorimétriques and some others recommended by the French Association for Standardization (AFNOR). The sampled stations revealed poor dissolved oxygens levels and present a state of eutrophisation.

Conclusion and application: This should draw the attention of local residents and political decisions on the management of sewage domestic, industrial and agricultural waste.

Key words: Physico-chemical quality, Ahémé Lake, eutrophisation, garbage.

préoccupation (Lamizana-Diallo et al, 2008). Les rejets d'eaux usées domestiques et agricoles même industrielle n'en demeurent pas non plus négligeables. Or ces lagunes saumâtres constituent des milieux de haute productivité biologique (Albaret, 1994 ; Baran, 1999) et de fortes possibilités de production halieutique, ce qui leur confère une 
grande importance économique. Cette pollution induit entre autres l'eutrophisation du système complet avec pour conséquences la mortalité des poissons et le développement de micro-organismes pathogènes néfastes pour l'environnement (Scheren

\section{MATÉRIELS ET MÉTHODES}

Milieu d'étude : Cette étude s'est déroulée sur la périphérie du lac Ahémé au Bénin (Afrique de l'ouest). Le lac Ahémé $\left(78 \mathrm{~km}^{2}\right)$ est dans un climat subéquatorial. II relie le fleuve Couffo et la lagune côtière de Ouidah dans le bassin hydrographique de Mono-Couffo. Autour de lui et al., 2004). Notre objectif est d'évaluer la qualité physico-chimique du Lac Ahémé au Bénin afin de connaître les risques possibles pour les espèces aquatiques et la santé humaine.

se développent l'agriculture, l'élevage. L'usine d'eau thermale de Possotomè y jette ses eaux usées peu/pas traitées. La pêche y est pratiquée avec la pratique abusive d'Acadja (piège aux poissons), suivi du transport fluvial par des barques souvent motorisées

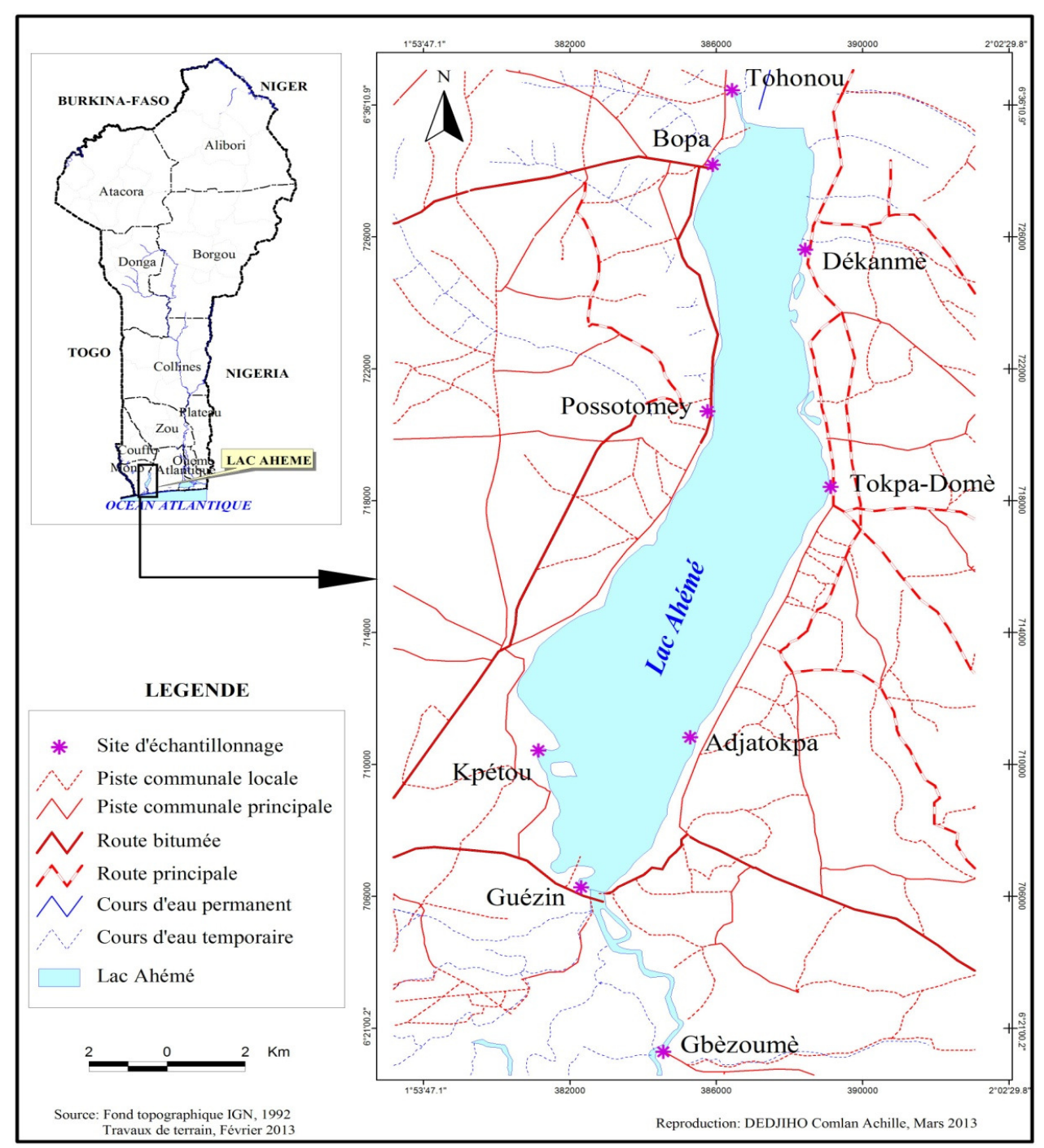

Figure 1 : Localisation de la zone d'étude 
Tableau 1 : Stations d'échantillonnage et raison du choix

\begin{tabular}{|c|c|c|}
\hline Stations d'échantillonnage & Raisons du choix & $\begin{array}{l}\text { Coordonnées } \\
\text { géographiques }\end{array}$ \\
\hline Tohonou & Réceptacle les eaux du fleuve Couffo & 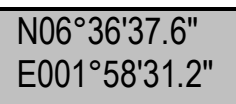 \\
\hline Bopa & $\begin{array}{l}\text { Réceptacle des déchets solides et liquides ménagers et } \\
\text { ceux provenant des salons de coiffures et garages des } \\
\text { mécaniciens. Il est lieu de lavage par moment. }\end{array}$ & 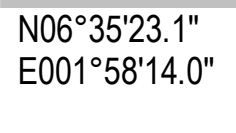 \\
\hline Possotomè & $\begin{array}{l}\text { Réceptacle des déchets liquides parfois mal/non traités } \\
\text { provenant de l'usine d'eau thermale de Possomè. }\end{array}$ & $\begin{array}{l}\text { N06 } 31^{\prime} 16.7^{\prime \prime} \\
\text { E001 }^{\circ} 58^{\prime} 09.7^{\prime \prime}\end{array}$ \\
\hline Kpétou & $\begin{array}{l}\text { Élevage de porcs, rejet d'ordures ménagères, défécation } \\
\text { des êtres humains sur la berge. }\end{array}$ & $\begin{array}{l}\mathrm{N} 06^{\circ} 25^{\prime} 31.9^{\prime \prime} \\
\mathrm{E} 001^{\circ} 55^{\prime} 17.0^{\prime \prime}\end{array}$ \\
\hline Guézin & Village semi-lacustre ; contact direct des WC avec le lac & $\begin{array}{l}\mathrm{N} 06^{\circ} 23^{\prime} 20.5^{\prime \prime} \\
\mathrm{E} 001^{\circ} 56^{\prime} 21.1^{\prime \prime}\end{array}$ \\
\hline Dékanmè & $\begin{array}{l}\text { Réceptacle des eaux de ruissellement contenant des } \\
\text { déchets des ménages et des intrants agricoles. }\end{array}$ & $\begin{array}{l}\mathrm{N} 06^{\circ} 33^{\prime} 58.5^{\prime \prime} \\
\mathrm{E} 001^{\circ} 59^{\prime} 37.6^{\prime \prime}\end{array}$ \\
\hline Tokpa-Domè & $\begin{array}{l}\text { Réceptacle des eaux de ruissellement contenant des } \\
\text { déchets des ménages et des intrants agricoles. }\end{array}$ & $\begin{array}{l}\mathrm{N} 06^{\circ} 30^{\prime} 01.2^{\prime \prime} \\
\mathrm{E} 002^{\circ} 00^{\prime} 00.9^{\prime \prime}\end{array}$ \\
\hline Adjatokpa & $\begin{array}{l}\text { Réceptacle des eaux de ruissellement contenant des } \\
\text { déchets des ménages et des intrants agricoles. }\end{array}$ & $\begin{array}{l}\mathrm{N} 06^{\circ} 25^{\prime} 50.6^{\prime \prime} \\
\mathrm{E} 001^{\circ} 57^{\prime} 54.9^{\prime \prime}\end{array}$ \\
\hline Gbèzoumè & $\begin{array}{l}\text { Réceptacle des eaux de ruissellement contenant des } \\
\text { déchets des ménages et des intrants agricoles. }\end{array}$ & $\begin{array}{l}\mathrm{N} 06^{\circ} 20^{\prime} 36.3^{\prime \prime} \\
\mathrm{E} 001^{\circ} 57^{\prime} 31.4^{\prime \prime}\end{array}$ \\
\hline
\end{tabular}

Collecte des données : L'échantillonnage a couvert une période de douze mois à raison d'une campagne d'échantillonnage par mois (novembre 2011 à octobre 2012), aux stations qui figurent dans le tableau 1.

Tableau 2 : Récapitulatif des paramètres mesurés et méthodes utilisées

\begin{tabular}{|c|c|}
\hline Paramètres étudiés & Appareils et Méthodes \\
\hline pH, Température, Oxygène dissous & $\begin{array}{l}\text { Mesure directe par le multiparamètre } \mathrm{pH} / \text { Oximètre WTW } \\
340 \mathrm{i} \text {, }\end{array}$ \\
\hline Conductivité, Salinité & $\begin{array}{l}\text { Mesure directe par le multiparamètre Conductimètre WTW } \\
340 \mathrm{i}\end{array}$ \\
\hline Matières en Suspension (MES), Couleur, Turbidité & Colorimètre HACH DR/890, Méthode 8025 \\
\hline Paramètres chimiques (Nitrates, Nitrites, phosphates) & $\begin{array}{l}\text { (Méthode spectrophotométrique HACH LANG DR5000) } \\
\text {-NitraVer®5 Nitrate Reagent for } 10 \mathrm{ml} \text { sample, Cat. 21061- } \\
69 \mathrm{Pk} / 100 \text {, } \\
\text {-NitriVer }{ }^{\circledR 2} \text { Nitrite Reagent for } 10 \mathrm{ml} \text { sample, Cat. } 21075- \\
69 \mathrm{Pk} / 100 \\
\text {-PhosVer } \\
21060 \text { Phosphate Reagent for } 10 \mathrm{ml} \text { sample, Cat. }\end{array}$ \\
\hline $\begin{array}{l}\text { Demande Biochimique en Oxygène au bout de cinq } \\
\text { jours }\left(\mathrm{DBO}_{5}\right)\end{array}$ & $\begin{array}{l}\text { Méthode respirométrique OxiTop dans une enceinte } \\
\text { thermostatée }\end{array}$ \\
\hline Demande Chimique Oxygène (DCO) & $\begin{array}{l}\text { Norme AFNOR NF T90-101, Colorimètre, Méthode de } \\
\text { Dichromate de potassium }\end{array}$ \\
\hline Grande Saison Sèche & Petite Saison Sèche \\
\hline Grande Saison Pluvieuse & Petite Saison Pluvieuse \\
\hline
\end{tabular}




\section{RÉSULTATS}

Les résultats sont présentés par les histogrammes suivants :

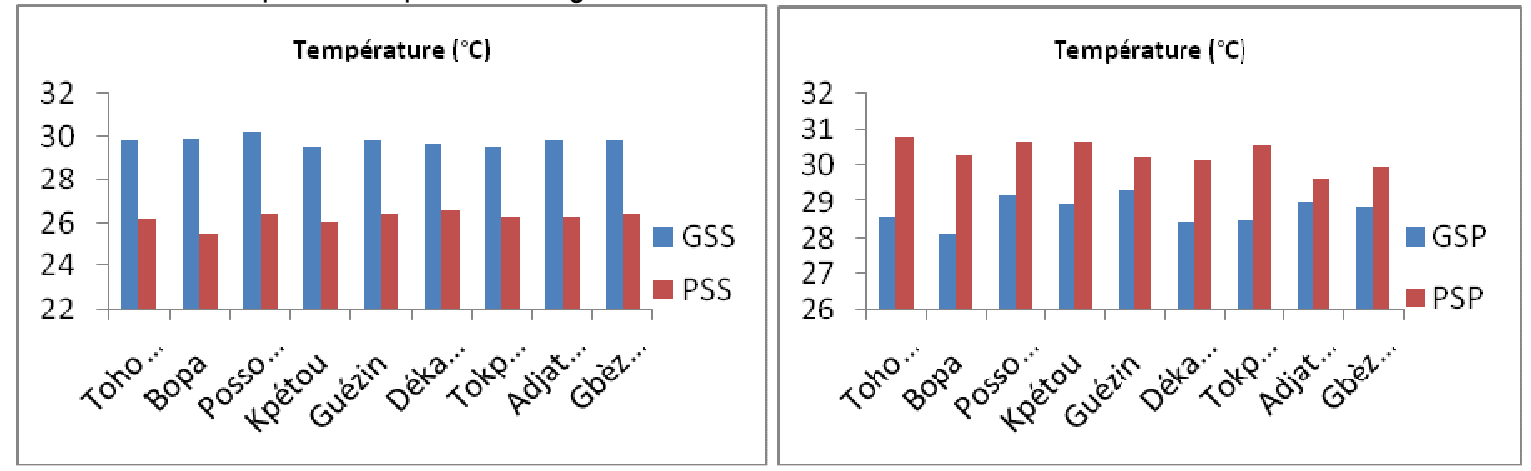

Figure 2: Variabilité de la température en fonction des stations d'échantillonnage

On constate que la température varie en moyenne de $26,24^{\circ} \mathrm{C}$ pour la PSS à $29,79^{\circ} \mathrm{C}$ pour la GSS d'une part et de $30,32^{\circ} \mathrm{C}$ pour la $\mathrm{PSP}$ à $28,77^{\circ} \mathrm{C}$ pour la GSP d'autre part.
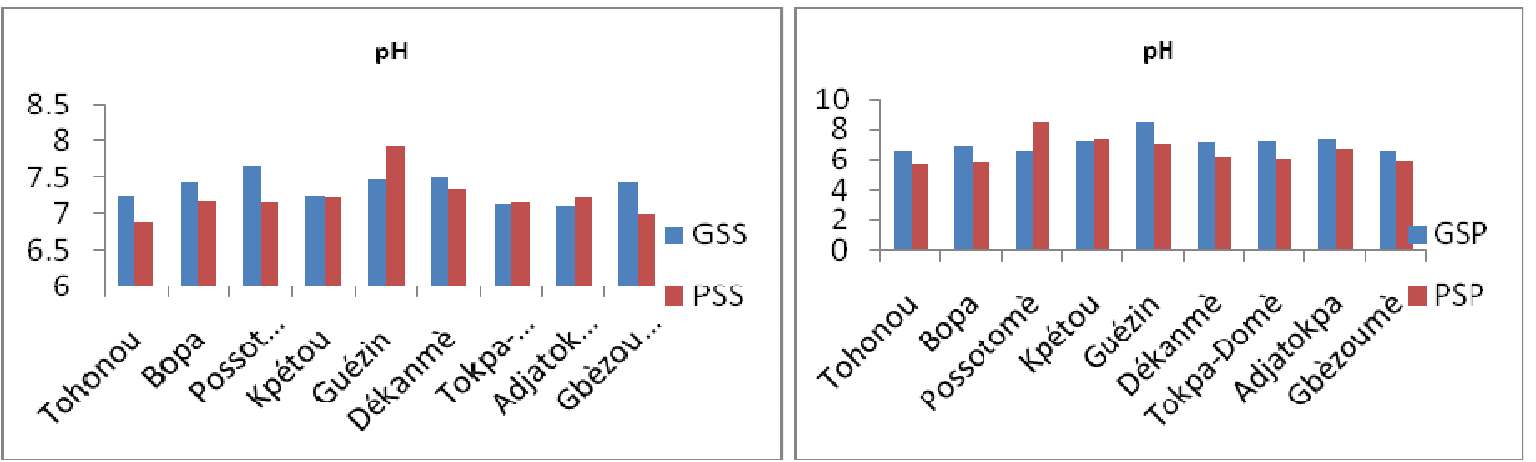

Figure 3 : Variabilité du pH en fonction des stations d'échantillonnage

Le pH quand à lui présente respectivement les moyennes 7,36 pour la GSS, 7,23 pour la PSS, 7,11 pour GSP et 6,53 pour la PSP.
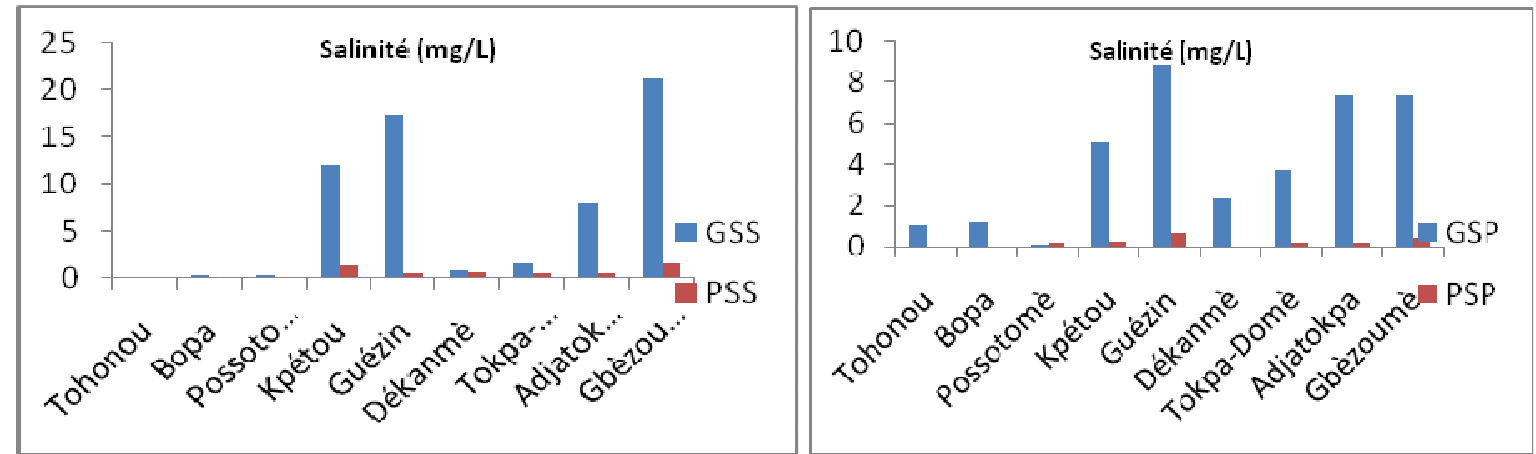

Figure 4 : Variabilité de la salinité en fonction des stations d'échantillonnage 


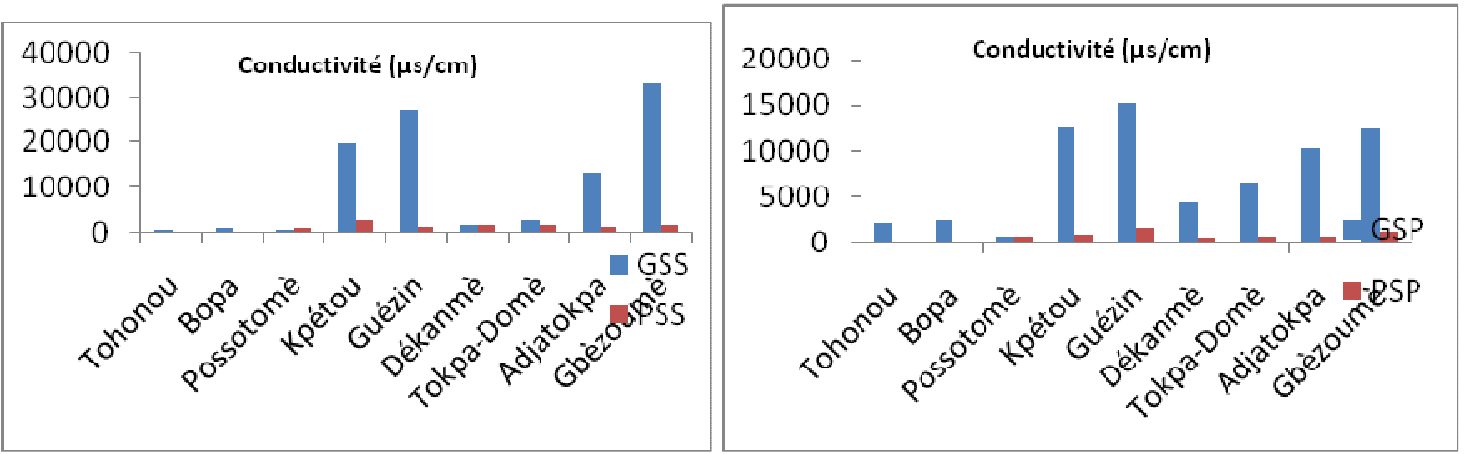

Figure 5 : Variabilité de la conductivité en fonction des stations d'échantillonnage

La salinité et la conductivité révèlent leurs valeurs les plus élevée au niveau des stations Kpétou, Guézin et Gbèzoumè.

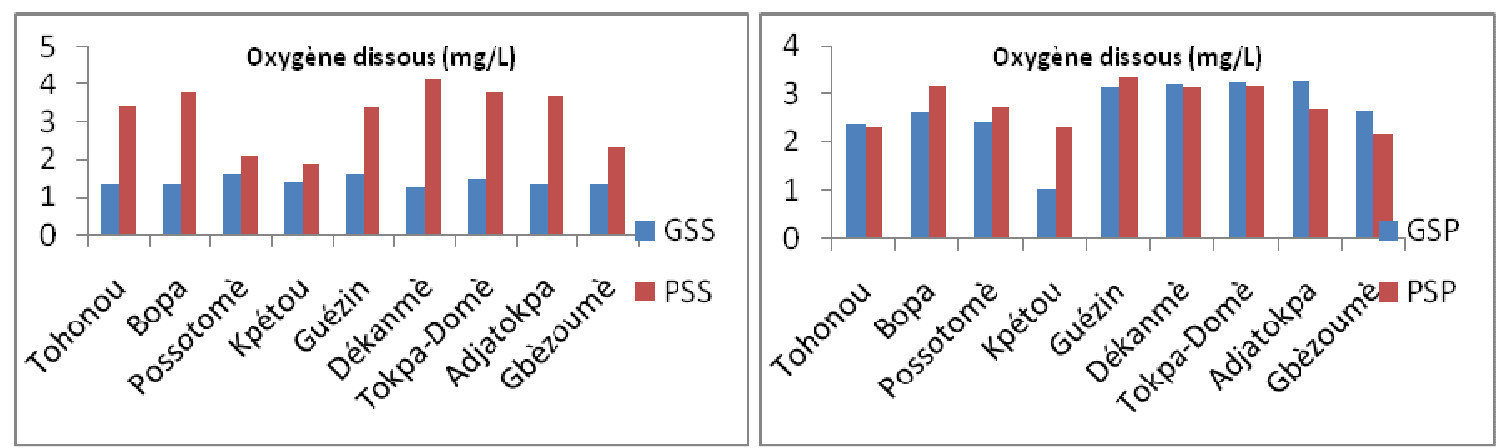

Figure 6: Variabilité de l'oxygène dissous en fonction des stations d'échantillonnage

Nous constatons que seule la GSS est la seule saison qui présente en majorité la teneur en oxygène dissous en dessous de $3 \mathrm{mg} / \mathrm{L}$.

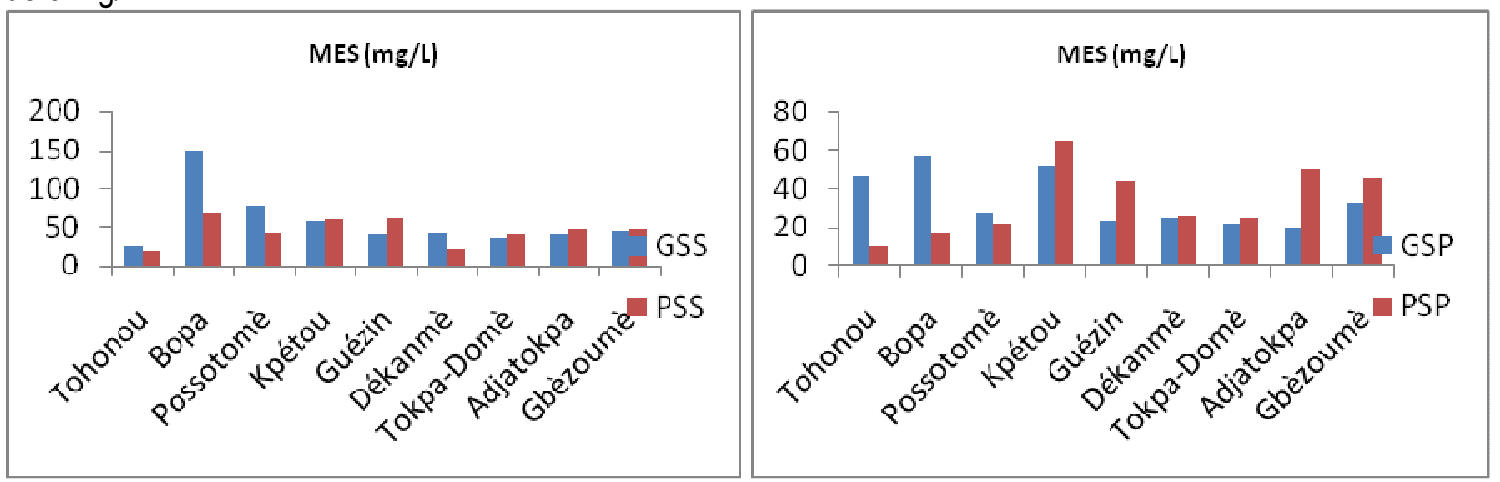

Figure 7 : Variabilité des MES en fonction des stations d'échantillonnage 

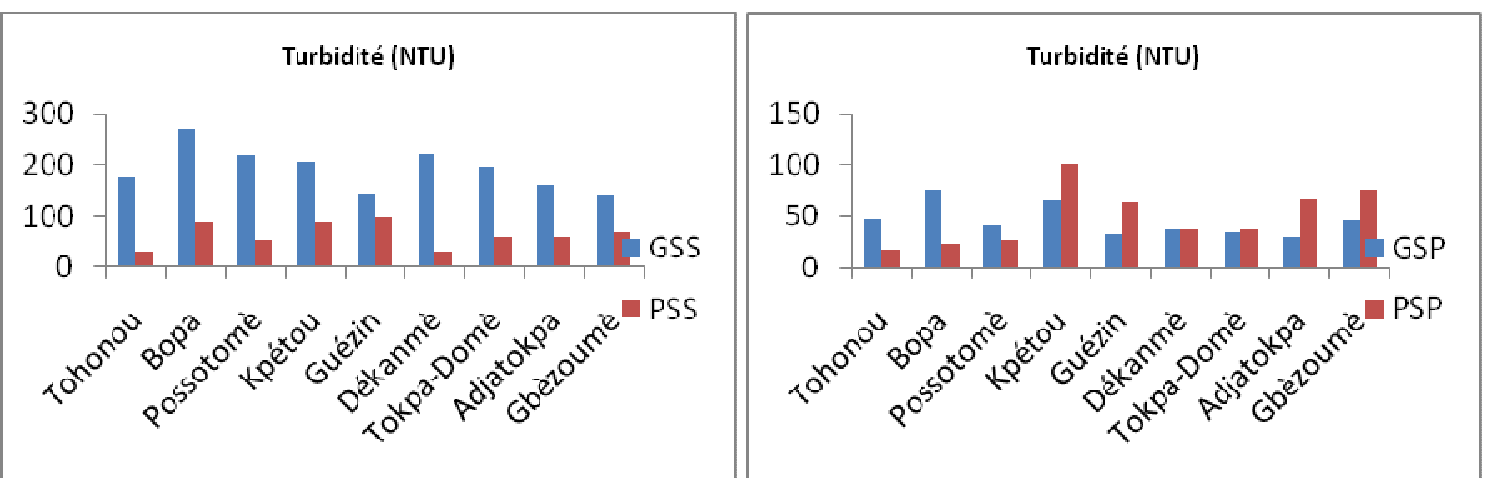

Figure 8 : Variabilité de la turbidité en fonction des stations d'échantillonnage
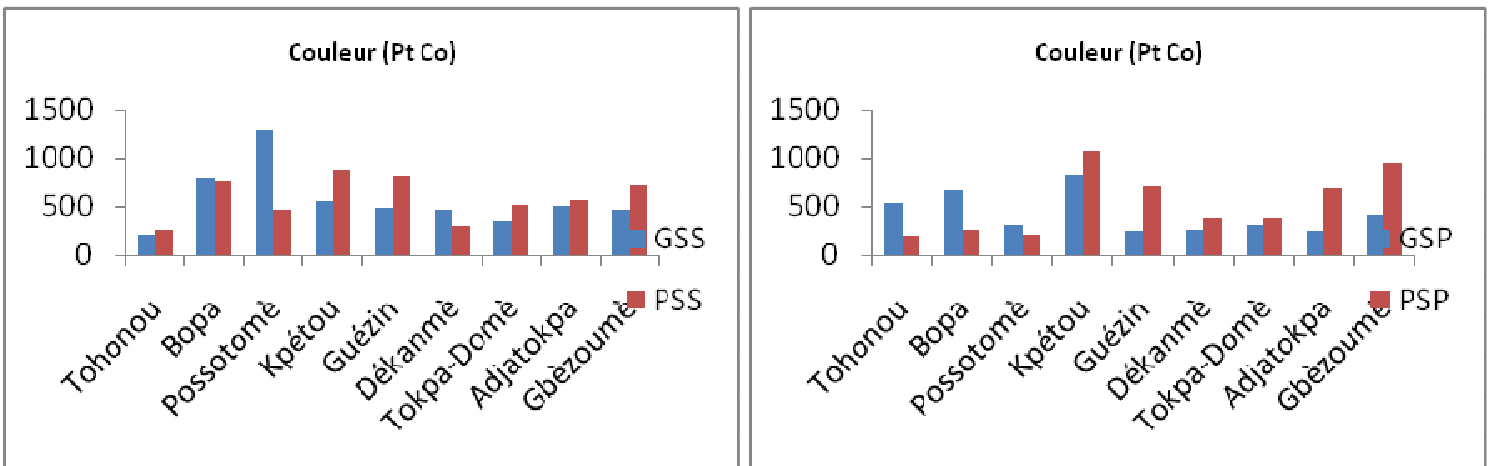

Figure 9 : Variabilité de la couleur en fonction des stations d'échantillonnage

Nous constatons que c'est aux saisons pluvieuses qu'il y a plus d'apports de MES, les plus grandes turbidités puis de la couleur, surtout au niveau des stations Kpossotomè, Kpétou et Guézin.

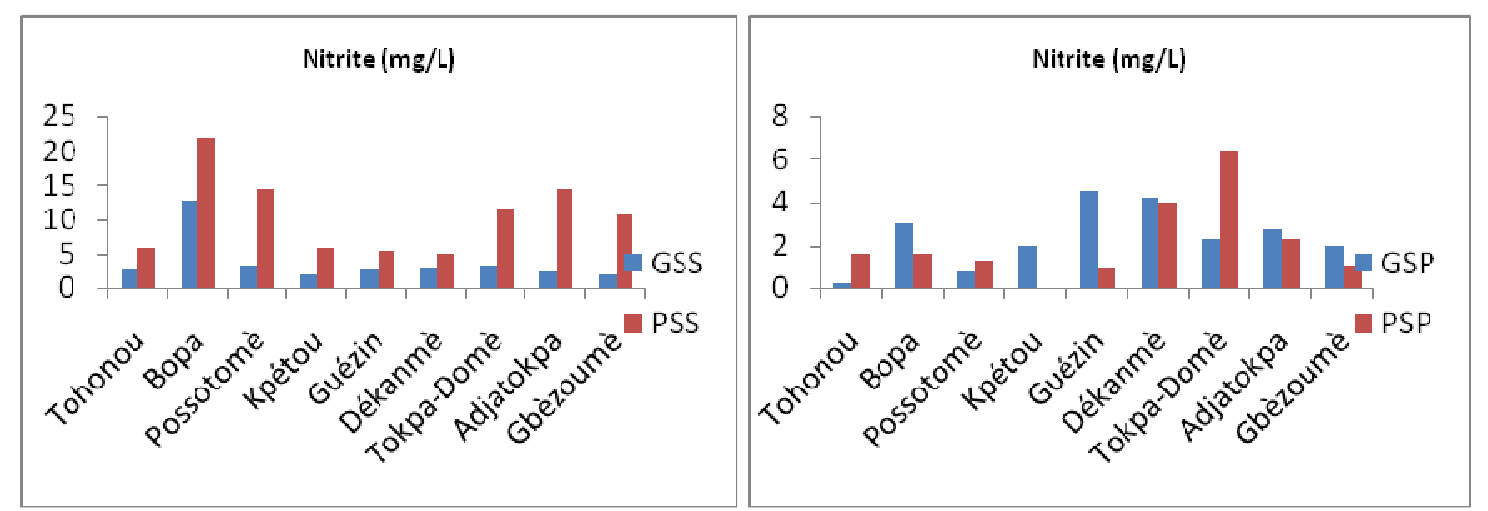

Figure 10 : Variabilité des ions nitrites en fonction des stations d'échantillonnage 

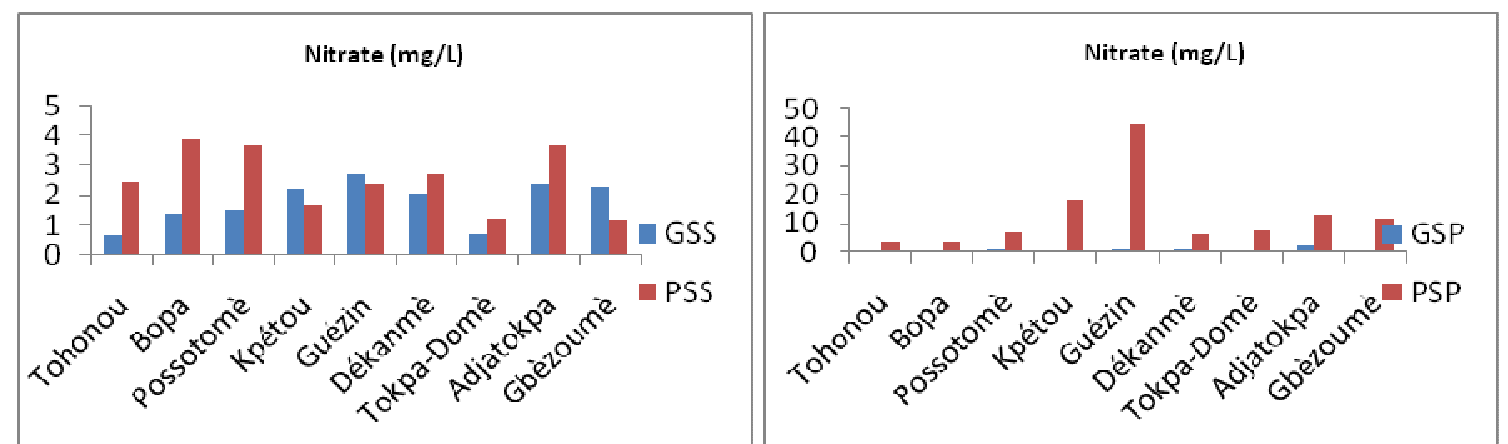

Figure 11 : Variabilité des ions nitratent en fonction des stations d'échantillonnage
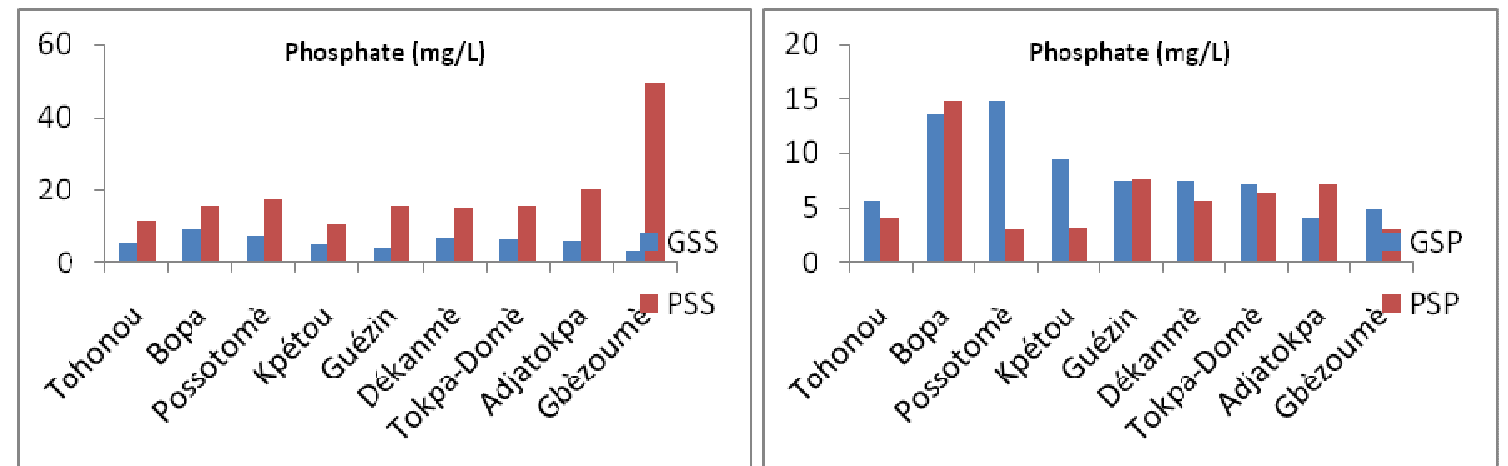

Figure 12 : Variabilité des ions phosphatent en fonction des stations d'échantillonnage

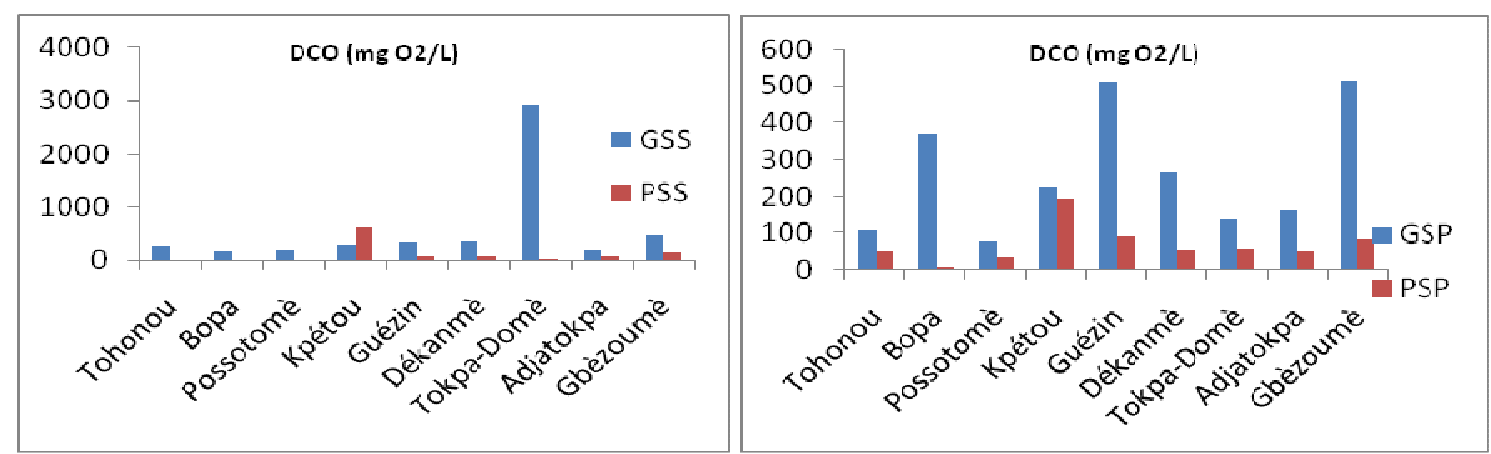

Figure 13 : Variabilité de la DCO en fonction des stations d'échantillonnage
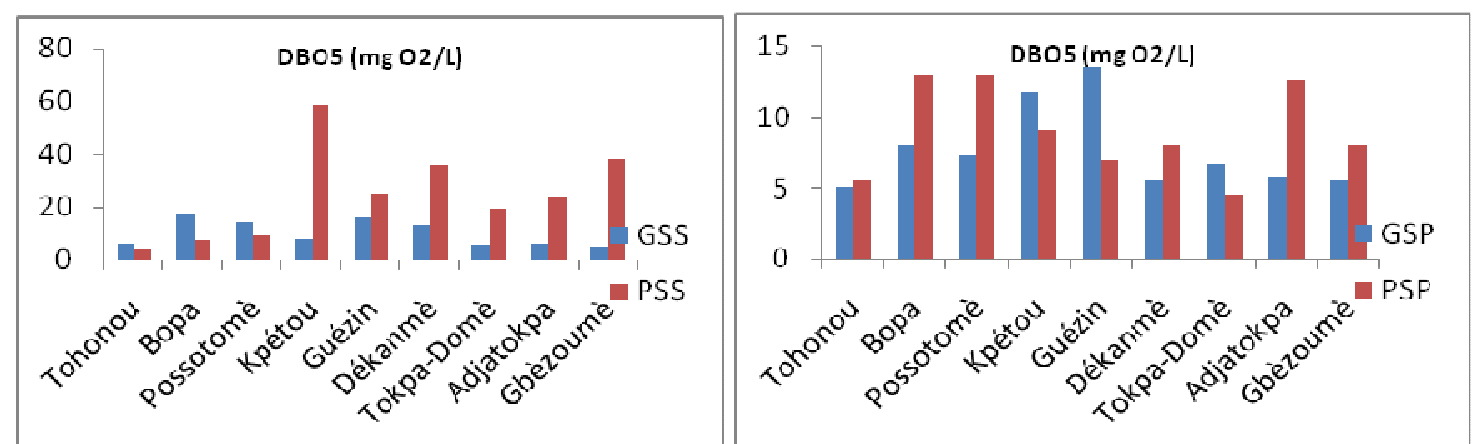

Figure 14 : Variabilité de la $\mathrm{DBO}_{5}$ en fonction des stations d'échantillonnage

Nous constatons que les valeurs les plus élevées de $\mathrm{DBO}_{5}$ ou de $\mathrm{DCO}$ sont obtenues au niveau des stations Kpétou, Guézin. 


\section{Dèdjiho et al J. Appl. Biosci. 2013. Évaluation de la qualité physico-chimique de tributaires d'eaux}

usées du Lac Ahémé, Benin

\section{DISCUSSION}

Les valeurs de la température enregistrées (figure 2), sont concordantes à celles de Dèdjiho (2011) et Poumon (1998) qui sont d'avis que les températures comprises entre 24 à $35^{\circ} \mathrm{C}$ sont favorables à une bonne croissance des espèces piscicoles couramment élevées. Egan et Boyd (1997) rapporté par Abou (2001) trouvent qu'une température de 28 à $32^{\circ} \mathrm{C}$ est optimale pour la croissance des tilapias. Par ailleurs, les valeurs de $\mathrm{pH}$ obtenues sont bonnes en aquaculture car situées entre 6,5 à 9 (Boyd, 1990 in Kanagire, 2001). Le lac s'alimente du fleuve Couffo et se déverse dans la mer, raison pour laquelle la salinité devient élevée lorsqu'on se rapproche de la mer. La montée des teneurs de la salinité et de la conductivité pendant la GSS au niveau des stations de Kpétou, Guézin Gbèzoumè (figures 4 et figure 6), s'explique par le phénomène de l'intrusion saline en marrée haute. Ces valeurs sont au dessus de celles trouvées par Guinda (1996) et Lamizana-Diallo et al (2008) qui ont travaillé respectivement sur Moumoune et Marsili au Burkina-Faso. La salinité a atteint à ces stations des concentrations supérieures à $7 \mathrm{~g} / \mathrm{L}$, limite audelà de laquelle elle peut inhiber la croissance des jacinthes d'eaux selon (Wilson et al, 2005). Les faibles teneurs en oxygène dissous ont été enregistrées à Kpétou et à Possotomè (figure 5) et, c'est aussi à ces stations qu'on a enregistré les fortes demandes chimiques et biochimiques en oxygène surtout en saisons sèches. . Selon Lamizana-Diallo et al. (2008), l'oxygène dissous est au carrefour de bons nombres de phénomènes. En effet, il intervient dans la respiration des organismes aquatiques, l'oxydation et la dégradation des polluants, l'activité photosynthétique de la flore et les échanges avec l'atmosphère. C'est ce qui explique la relation étroite qui existe entre eux. Selon Beaux (1998), une eau est qualifiée de polluée si elle a une teneur en

\section{CONCLUSION}

L'étude que nous venons de mener nous a permis de suivre l'évolution des paramètres physico-chimiques sur la périphérie du lac Ahémé afin d'évaluer les apports des eaux qui s'y jettent. II en ressort la dégradation de la qualité du lac : l'eutrophisation avec ses conséquences

\section{RÉFÉRENCES BIBLIOGRAPHIQUES}

Abou Y, 2001. Effet d'une couverture d'Azolla LAM sur la production piscicole du tilapia Oreochromis niloticus (L.) en étangs dans la banlieue de Cotonou (Bénin). Mémoire présenté pour l'obtention du diplôme d'études complémentaire en éco-technologie des eaux continentales. oxygène dissous inférieure à $3 \mathrm{mg} / \mathrm{l}$. La faible concentration en oxygène dissous serait aussi liée à la forte turbidité, au MES (figures 7 et 8). En effet, l'abondance des MES dans l'eau favorise la réduction de la luminosité et abaisse la production biologique du fait de la chute de l'oxygène dissous consécutive à une réduction des phénomènes de photosynthèse (LamizanaDiallo et al. (2008). Les fortes teneurs des DCO et $\mathrm{DBO}_{5}$ obtenues en aval (figures 13 et 14) sont dues aux forts rejets d'eaux usées directement dans le cours d'eau. On peut évoquer le cas des rejets d'eaux usées de l'usine de Possotomè, la proximité ou l'inexistence des latrines à Kpétou et du village semi-lacustre de Guézin. Selon Lamizana-Diallo (2005), cet état de pollution n'affecte pas la répartition des hygrophytes le long du bief du Massili mais, a un effet très négatif sur les poissons. Selon la grille de lecture de la colonne d'eau établie par l'institut français pour la recherche de la mer (ifremer, 2000), les teneurs en ions nitrites, nitrates et phosphates sont dans la gamme mauvaise signalant que le lac Ahémé est eutrophe. L'une des conséquences de l'eutrophisation est la prolifération des plantes aquatiques. En effet, la prolifération des plantes aquatiques surtout celle de la jacinthe d'eau est à l'origine de la formation d'un écran en surface des eaux qui empêche l'oxygénation du milieu Mama (2010). De plus leur décomposition induit une augmentation de la quantité de matière organique dans le milieu dont la dégradation favorise asphixie des espèces halieutiques. La production de sulfure et de nitrite (toxiques) inhibe le développement d'autres producteurs primaires et des poissons à grande sensibilité. Ceci peut provoquer un déséquilibre de la chaîne trophique (production/ consommation) d'un plan d'eau et entraîner des conséquences écologiques importantes.

multiples et parfois irréversibles tant pour les espèces halieutiques que pour les espèces humaines. Une attention particulière et une politique de gestion d'eaux usées et déchets doivent être menées afin de protéger nos plans d'eau de surface.

Facultés Universitaires Notre Dame de la Paix. Faculté des Sciences. Namur-Belgique. 85p.

Albaret JJ, 1994. Les poissons: Biologie et peuplements, p 239-273. In J.-R. Durand et al., (Eds.), Environnement et ressources aquatiques de 
Côte d'Ivoire. Tome II -Les milieux lagunaires. Op. cit.

Baran E, 1999. Rôle des estuaires vis à vis de la ressource halieutique côtière en Guinée, p.137157. In F. Domain, P. Chavance et A. Diallo (Eds.), La pêche côtière en Guinée : ressources et exploitation. Op. cit.

Beaux JF, 1998. L'environnement repères pratiques. Nathan, ISBN 209-18243-3, pp. 64-71.

Chang $\mathrm{H}, 2005$. Spatial and temporal variations of water quality in the Han River and its tributaries, Seoul, Korea. 1993-2002. Water, Air, and Soil Pollution 161, 267-284.

Dèdjiho C A, 2011. Évaluation de la chaine trophique d'une aire marine protégée en relation avec sa physico-chimie : cas de Gbèzoumè dans la commune de Ouidah. Mémoire de DEA. FAST/UAC, Bénin.

Guenda W, 1996. Étude faunistique, écologique et de la distribution des insectes d'un réseau hydrographique de l'Ouest africain : le Mouhoun (Burkina Faso) ; rapport avec Simulium damnosum Theobald, vecteur de l'onchocercose. Thèse d'État, Université de droit, d'économie et des sciences d'Aix Marseille III, pp. 13-50.

He H, Zhou J, Wu Y, Zhang W, Xie X, 2007. Modelling the response of surface water quality to the urbanization in Xi'an, China. Journal of Environmental Management (2007), doi:10.1016/j.jenvman.2006.12.043; In Press.

Ifremer, 2000. Mise à jour d'indicateurs du niveau d'eutrophisation des milieux lagunaires méditerranéens - Rapport final-09-2000, (Tome I - 236p) (tome II - 196p).

Kanangire C K, 2001. Effet de l'alimentaire des poisons avec Azolla sur l'écosystème agro-piscicole au Rwanda. Dissertation présentée en vue de l'obtention du grade de Docteur en sciences. Faculté Universitaire Notre Dame de la Paix. Faculté des sciences. Namur-Belgique. 220p.

Lacaze J C, 1996. L'eutrophisation des eaux marines et continentales, Ellipses, 191p.

Lalèyè P A, 1995. Écologie comparée de deux espèces de Chrisichthys, poissons siluriformes (Claroteidae) du complexe lagunaire lac Nokoué-lagune de Porto-Novo au Bénin. Thèse de Doctorat en Sciences, Université de Liège (Belgique), 152 p. + Annexes.

Lamizana-Diallo M. B., 2005. Effet du régime de la crue naturelle fluviale sur les hygrophytes. Cas d'un bief du Massili-Burkina Faso. Mémoire de DEA. Laboratoire de biologie et d'écologie végétales. UFR-SVT, Université de Ouagadougou. 77p.

Lamizana-Diallo M B, Kenfack S et Millogo-Rasolodimby $\mathrm{J}, 2008$. Évaluation de la qualité physicochimique de l'eau d'un cours d'eau temporaire du Burkina Faso - Le cas du Massili dans le Kadiogo. Sud Sciences et technologiques. ISSN 0796-5419 du 16 juin 2008.

Mampouya M., Kenfack S. et Cisse G., 2000. Contribution à la gestion environnementale des déchets industriels : Étude d'un système rustique d'épuration et de revalorisation des eaux usées industrielles en milieu tropical. Actes du colloque international Eau-Environnement et Santé Ouaga 2000, du 24 au 26 décembre 2000 à Ouagadougou, pp 76-78.

Ministère de l'environnement du Québec, 2001. Critères de qualité de l'eau de surface au Québec, document disponible sur : www.menv.gouv.qc.ca.

Paliwal R, Sharma P, Kansal A, 2007. Water quality modelling of the river Yamuna (India) using Qualée-Uncas: Journal of Environmental Management 83 (2007) 131-144.

Pouomogne V, 1998. Pisciculture en Milieu Tropical Africain. Comment produire du poisson à coût modéré. Presse Universitaire d'Afrique, Yaoundé. 263p.

Ramade F, 1982. Éléments d'écologie- écologie appliquée- action de l'homme sur la biosphère. McGraw Hill, Paris, ISBN 2- 7040-1039-x. pp 140-436.

Scheren P A G M, Kroeze C, Janssen F J J G, Hordijk L, Ptasinski K J, 2004. Integrated pollution assessment of the Ebrié Lagoon, Ivory Coast, West Africa. Journal of Marine water System 44 (1-2).

Wilson JR, Holst N, Rees M, 2005. Determinants and patterns of population growth in water hyacinth. Aquat. Bot. 81, p. 51-67 